\title{
The Materials Genome Initiative at the National Science Foundation: A Status Report after the First Year of Funded Research
}

\section{Kathleen Feldman and Sean R. Agnew}

\section{Introduction}

Materials research and development have been singled out by the current U.S. presidential administration as a high priority for support. In 2011, President Barack Obama announced the Materials Genome Initiative $(\mathrm{MGI})^{1}$ as an effort to accelerate the pace of materials research, with specific goals of cutting in half both the cost and time to bring a new material to market (Figure 1). This vision will require moving beyond the traditional linear progression of fundamental research and discovery through development and deployment. New materials will instead come to market through an iterative process, with feedback at each step of the way facilitated by a materials innovation infrastructure founded on highquality digital data and advanced experimental and computational tools.
The MGI is being supported by activities across the federal research complex, with funding from the Department of Defense (DoD), Department of Energy (DoE), National Institute for Standards and Technology (NIST), and the National Science Foundation (NSF). The NSF is participating in MGI through the Designing Materials to Revolutionize and Engineer our Future (DMREF) program, ${ }^{2}$ with involvement of the Engineering, Math, and Physical Sciences, as well as the Computer and Information Science Directorates. With an emphasis on NSF's role in supporting fundamental research, the grants are intended to bring together experiment, theory, and computation in a tight iterative loop in which computation suggests avenues of exploration employing experiments and theory, while experiments and theory are, in turn, used to validate computation. The first round of DMREF awards was announced in September 2012, and 14 projects were funded, representing a total investment of approximately $\$ 13$ million. Nearly all of the projects are collaborative, involving at least three co-principle investigators. Several include industrial collaborators and are co-funded through the NSF GOALI $^{3}$ (Grant Opportunities for Academic Liaison with Industry) solicitation, which promotes university-industry partnerships.

\section{The DMREF Grantees Workshop}

Recognizing the importance of supporting this nascent community of researchers, NSF hosted a DMREF grantees workshop in September 2013 in Arlington, Virginia. While the funded research projects represent a wide spectrum of materials, they
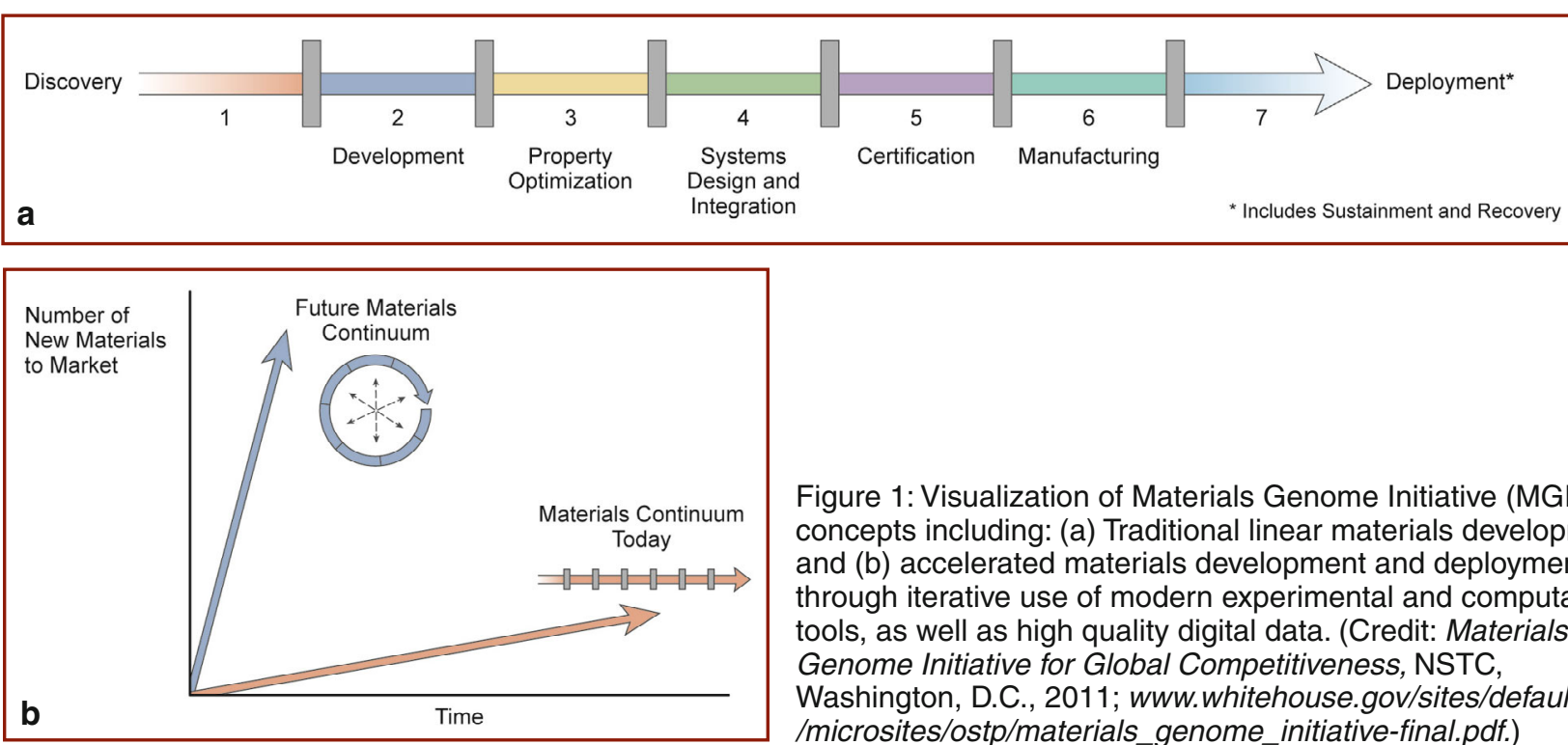

Figure 1: Visualization of Materials Genome Initiative (MGI) concepts including: (a) Traditional linear materials development and (b) accelerated materials development and deployment through iterative use of modern experimental and computational tools, as well as high quality digital data. (Credit: Materials Genome Initiative for Global Competitiveness, NSTC, Washington, D.C., 2011; www. whitehouse.gov/sites/default/files /microsites/ostp/materials_genome_initiative-final.pdf.) 
are both unified and challenged by the DMREF call to deeply integrate computation, experiment, and theory in meaningful collaborations. The workshop provided a face-to-face forum to strengthen ties within each project team and to provide opportunities for each of the teams to share their research progress with the others, as well as with program managers from across the federal research complex. The program also served to highlight areas that represent the greatest obstacles and opportunities for establishing and maintaining tightly integrated DMREF research programs. Each of the 14 initial DMREF projects was represented by at least one researcher. Some brought teams of researchers to capitalize on the opportunity to have face-to-face discussions.

In addition to technical updates by the teams, plenary presentations by Anthony Rollett, Carnegie Mellon University, and Surya Kalidindi, Georgia Institute of Technology, set the stage for discussions among the participants of broader issues common to many materials researchers, particularly at the fundamental level. Both of these plenary speakers provided their perspective on a related and up-and-coming area of research, mesoscale modeling in materials science. Rollett emphasized that the models for single crystal behavior are well developed and that continuum level models are also well in hand, while mesoscale models that link the two generally fail to well-describe the complex microstructure-dependent properties.

In particular, Rollet provided a helpful list of the state of modeling in materials science, albeit with some bias toward structural materials. He stated during his talk, "There is a rough hierarchy of materials properties according to the reliability and ease of calculation from first principle, such as [electron density functional theory] or DFT, combined with microstructure, for example:

- Accepted: elastic moduli, most single crystal properties (i.e. no microstructure)

- Feasible: yield strength in metals

- Challenging: strain hardening, ductility, fatigue, thermal

Table I. A List of the First Cohort of Projects Sponsored by the NSF: Designing Materials to Revolutionize and Engineer our Future (DMREF) Program

\begin{tabular}{|c|c|c|}
\hline DMREF Project Title & Principal Investigators & Research Institution \\
\hline $\begin{array}{l}\text { * Collaborative Research: Nitride Discovery—Creating the } \\
\text { Knowledge Base for Hard Coating Design }\end{array}$ & $\begin{array}{l}\text { Sanjay Khare } \\
\text { Daniel Gall }\end{array}$ & $\begin{array}{l}\text { University of Toledo } \\
\text { Rensselaer Polytechnic Institute }\end{array}$ \\
\hline $\begin{array}{l}\text { GOALI - Discovery, Development, and Deployment of } \\
\text { High Temperature Coating/Substrate Systems }\end{array}$ & Tresa Pollock & $\begin{array}{l}\text { University of California- } \\
\text { Santa Barbara }\end{array}$ \\
\hline $\begin{array}{l}\text { Collaborative Research: Multi-Scale Modeling and Characterization } \\
\text { of Twinning-Induced Plasticity and Fracture in Magnesium Alloys }\end{array}$ & $\begin{array}{l}\text { Sean Agnew } \\
\text { Haitham El Kadiri } \\
\text { Mohammed Cherkaoui }\end{array}$ & $\begin{array}{l}\text { University of Virginia } \\
\text { Mississippi State University } \\
\text { Georgia Institute of Technology }\end{array}$ \\
\hline $\begin{array}{l}\text { * Collaborative Research: Multi-Scale Fundamental } \\
\text { Investigation of Sintering Anisotropy }\end{array}$ & $\begin{array}{l}\text { Rajendra Bordia } \\
\text { Eugene Olevsky }\end{array}$ & $\begin{array}{l}\text { University of Washington } \\
\text { San Diego State University }\end{array}$ \\
\hline $\begin{array}{l}\text { GOALI: High Efficiency Hierarchical Thermoelectric Composites } \\
\text { by Multiscale Materials Design and Development }\end{array}$ & Jihui Yang & University of Washington \\
\hline $\begin{array}{l}\text { Collaborative Research: Enhanced Functionalities in } 5 d \text { Transition- } \\
\text { Metal Compounds from Large Spin-Orbit Coupling }\end{array}$ & $\begin{array}{l}\text { David Vanderbilt } \\
\text { Janice Musfeldt }\end{array}$ & $\begin{array}{l}\text { Rutgers University } \\
\text { University of Tennessee }\end{array}$ \\
\hline $\begin{array}{l}\text { *Collaborative Research: First-Principles Based Design of } \\
\text { Spintronic Materials and Devices }\end{array}$ & $\begin{array}{l}\text { William Butler } \\
\text { Avik Ghosh }\end{array}$ & $\begin{array}{l}\text { University of Alabama, Tuscaloosa } \\
\text { University of Virginia }\end{array}$ \\
\hline Engineering Organic Glasses & Lian Yu & University of Wisconsin-Madison \\
\hline $\begin{array}{l}\text { High-Pressure Synthesis of Novel Oxynitride Photocatalysts } \\
\text { Directed by Theory and In Situ Scattering }\end{array}$ & John Parise & SUNY at Stony Brook \\
\hline Multifunctional Interfacial Materials by Design & Chang-Beom Eom & University of Wisconsin-Madison \\
\hline $\begin{array}{l}\text { *A Fundamental Approach to Study the Effect of Structural and } \\
\text { Chemical Composition in Functionalized Graphene Materials }\end{array}$ & Horacio Espinosa & Northwestern University \\
\hline $\begin{array}{l}\text { GOALI: Computational and Experimental Discovery and Development } \\
\text { of Additives for Novel Polymer Morphology and Performance }\end{array}$ & Gregory Rutledge & Massachusetts Institute of Technology \\
\hline $\begin{array}{l}\text { *Collaborative Research - Programmable Peptide-Based } \\
\text { Hybrid Materials }\end{array}$ & $\begin{array}{l}\text { Darrin Pochan } \\
\text { Jeffery Saven }\end{array}$ & $\begin{array}{l}\text { University of Delaware } \\
\text { University of Pennsylvania }\end{array}$ \\
\hline $\begin{array}{l}\text { Collaborative Research: Simulation-Based Design of Functional } \\
\text { Sub-nanometer Porous Membranes }\end{array}$ & $\begin{array}{l}\text { Sinan Keten } \\
\text { Ting Xu }\end{array}$ & $\begin{array}{l}\text { Northwestern University } \\
\text { University of California-Berkeley }\end{array}$ \\
\hline
\end{tabular}

* denotes those projects highlighted in the sidebars of this article 
conductivity, electrical conductivity, hydrogen embrittlement

- [Very challenging]: corrosion, liquid metal embrittlement, joining processes (welding) Real materials must meet multiple requirements for their properties.

Kalidindi provided a complementary perspective that emphasized the stochastic nature of materials microstructures and resultant microstructure-dependent properties. He emphasized the need for "a major transformation (including a culture change) in materials design and development protocols," in order to achieve the goals of the MGI. Kalidindi also gave specific illustrations of the use of data mining techniques to explore the incredibly large, interrelated, and multi-dimensional spaces of materials composition, processing, microstructure, and properties.

He demonstrated that even simple data mining concepts like principle component analysis (PCA) can be powerful tools for discriminating between microstructures and for determining structure-property connections. ${ }^{4}$ It was acknowledged by attendees that few materials scientists are even aware of these approaches.

\section{Status of the DMREF Program}

Despite the modest size of the program (approximately $\$ 13$ million allocated to the first cohort of projects, with an average funding of $\$ 280$ thousand per project per year over 3-4 years), DMREF projects represent a broad range of material classes, from designed peptides and proteins, to functional/electronic/spintronic crystals and films, to structural metals

\section{DMREF Technical Highlight Nitride Discovery: Creating the Knowledge Base for Hard Coating Design}

Sanjay V. Khare, University of Toledo, and Daniel Gall, Rensselaer Polytechnic Institute, represent one of the smaller teams, yet they clearly embody the MGIDMREF vision of a tightly woven interaction between computation (Khare) and experimentation (Gall). A worldwide research and development effort has yielded steady, incremental improvements in the performance of wear and corrosion protective transition metal nitride coatings over the past three decades. However, the introduction of new nitrides has been hampered by missing knowledge on basic materials properties for most of the 30 binary nitrides, and by the complexity of simultaneously optimizing composition, microstructure, and processing. Computational prediction of mechanical properties and oxidation resistance has

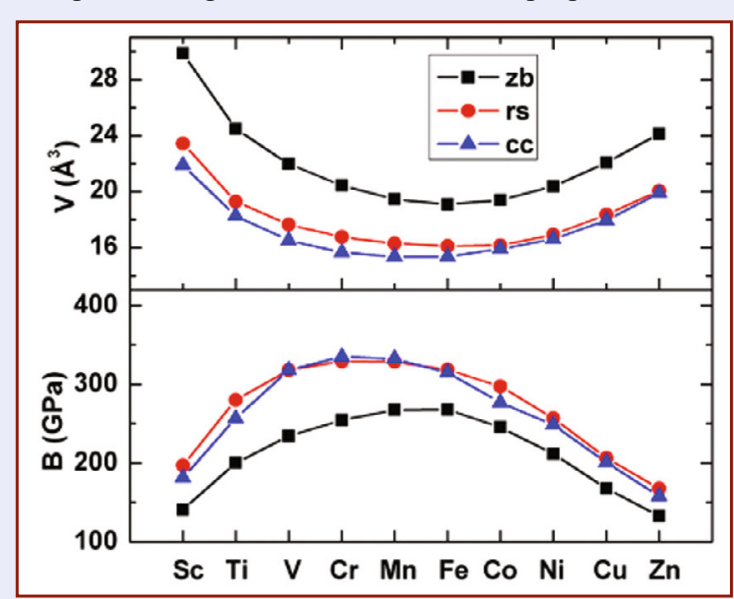

already begun to result in a quantitative understanding of the fundamental properties for all transition metal nitrides (Figure A), and a large database of such basic properties is under development for all for cubic nitrides. Promising candidates are being fabricated by thin film deposition techniques.

Figure A. Structure (unit cell volume) property (Bulk modulus $=(\mathrm{C} 11+2 \mathrm{C} 12) / 3$ relationships predicted from first principles modeling of zinc blende (zb), rock salt (rs) and cesium chloride (cs) structured ceramics. ${ }^{8}$ (@ IOP Publishing. Reproduced by permission of IOP Publishing. All rights reserved.) and ceramics (Table I). Within every project, the program emphasizes tight integration between experiment, theory, and computation. Results from each element are expected to feed back into the others in an iterative loop, so that computational models are validated by the most advanced and accurate experimental data, and experiments are directly informed and guided by simulations and theory. As such, DMREF projects are pushing the boundaries of materials computation across a wide range of materials, both in areas with wellintegrated computational methods, ${ }^{5}$ such as structural metals and composites, as well as those in which computation is yet underutilized, as in soft materials.

\section{Structural Materials and Coatings}

The DMREF program supports several projects in this area, and in the field of structural materials, the computational tools that people imagine employing within MGI research are beginning to become well-integrated into the research workflow. At the lowest length scales, density functional theory (DFT) codes such as VASP $^{6}$ and FLAPW 7 are widely employed within largescale alloy/material development programs. One of the smaller-in terms of the number of co-principal investigators-DMREF projects represents a complete materials development, from first principles modeling to experimental synthesis and testing. This team is exploring the large compositional space of potential cubic nitrides for protective coatings (see sidebar).

First principles modeling is often employed to determine phase stability data as input to thermodynamic modeling tools, such as ThermoCalc, ${ }^{9}$ Pandat, ${ }^{10}$ and FACTSage ${ }^{11}$ which are employed in the calculation of phase diagrams and solidification pathways. At the atomistic lengthscale, numerous molecular dynamics codes, including open source codes such as LAMMPS, ${ }^{12}$ are available for atomistic simulations. The interatomic 
potentials employed within such codes are increasingly parameterized by fitting to DFT results, particularly for new materials developments, where the required physical property data is not yet available. At the highest length-scales, required to embody the integrated computational materials engineering (ICME) approach endorsed by the National Academy of Engineers, ${ }^{13}$ commercial and home-built finite element method (FEM) codes for structural and multi-physics modeling abound. Development of codes for simulating component and full structure level properties is generally not the domain of materials chemists, physicists, or engineers. However, finite element codes are increasingly employed by materials scientists for mesoscale research questions.

As mentioned previously, the area of mesoscale modeling, designed to link the lower scales to the continuum, is less codified. For instance, the phase field approaches that involve finding solutions to partial differential equations of $2^{\text {nd }}$ (Allen-Cahn) or $4^{\text {th }}$ (CahnHilliard) order, are, in turn, coupled with elasticity, heat transfer, and Navier-Stokes equations to study solid state phase transformations and solidification. Currently, researchers most frequently employ completely home-built codes or employ commercial codes only for certain elements, such as solving the differential equations using finite difference or finite element methods. As far as commercialization is concerned, the authors are only aware of a single dedicated phase field modeling code known as MICRESS ${ }^{14}$ developed in Germany. None of the current cohort of DMREF projects employs phase field modeling as a central aspect of their approach. However, one area to which phase field modeling is beginning to be applied is powder processing of ceramics. One of the DMREF projects specifically focused on improving ceramic processing (see sidebar) makes use of continuum (finite element) and mesoscale (kinetic Monte Carlo) modeling elements.

Also at the mesoscale, discrete dislocation dynamics (DDD) codes are also generally home-built research tools, although the ParaDis ${ }^{15}$ code developed at Lawrence Livermore National Laboratory is available for download. Crystal plasticity modeling tools are in a similar state of development: VPSC ${ }^{16}$ and variations of a UMAT ${ }^{17}$ for ABAQUS (Dassault Systémes, Waltham, Massachusetts) are prime examples of the research tools being employed today.

\section{Electronic/Functional Materials}

A significant challenge in functional materials stems from the vast composition space to be explored. ${ }^{18}$ At the atomistic level, calculations of electronic structure and related properties are done

\section{DMREF Technical Highlight Multi-Scale Fundamental Investigation of Sintering Anisotropy}

A team led by Rajendra Bordia, Clemson University, is developing a new, multi-scale theory of particulate sintering that takes into account microstructural anisotropy. The methodology is a step towards a full-fledged, multi-scale virtual reality - supported and verified by fundamental experiments - of the sintering of powder materials (Figure B). The work will provide a practical approach to design and optimize the manufacturing of advanced ceramic and metal systems with programmable macroscopic characteristics and micro-structure. The approach will be useful in understanding and optimizing densification and microstructural control in complex sintering situations, including processing of multi-layered systems like solid oxide fuel cells.
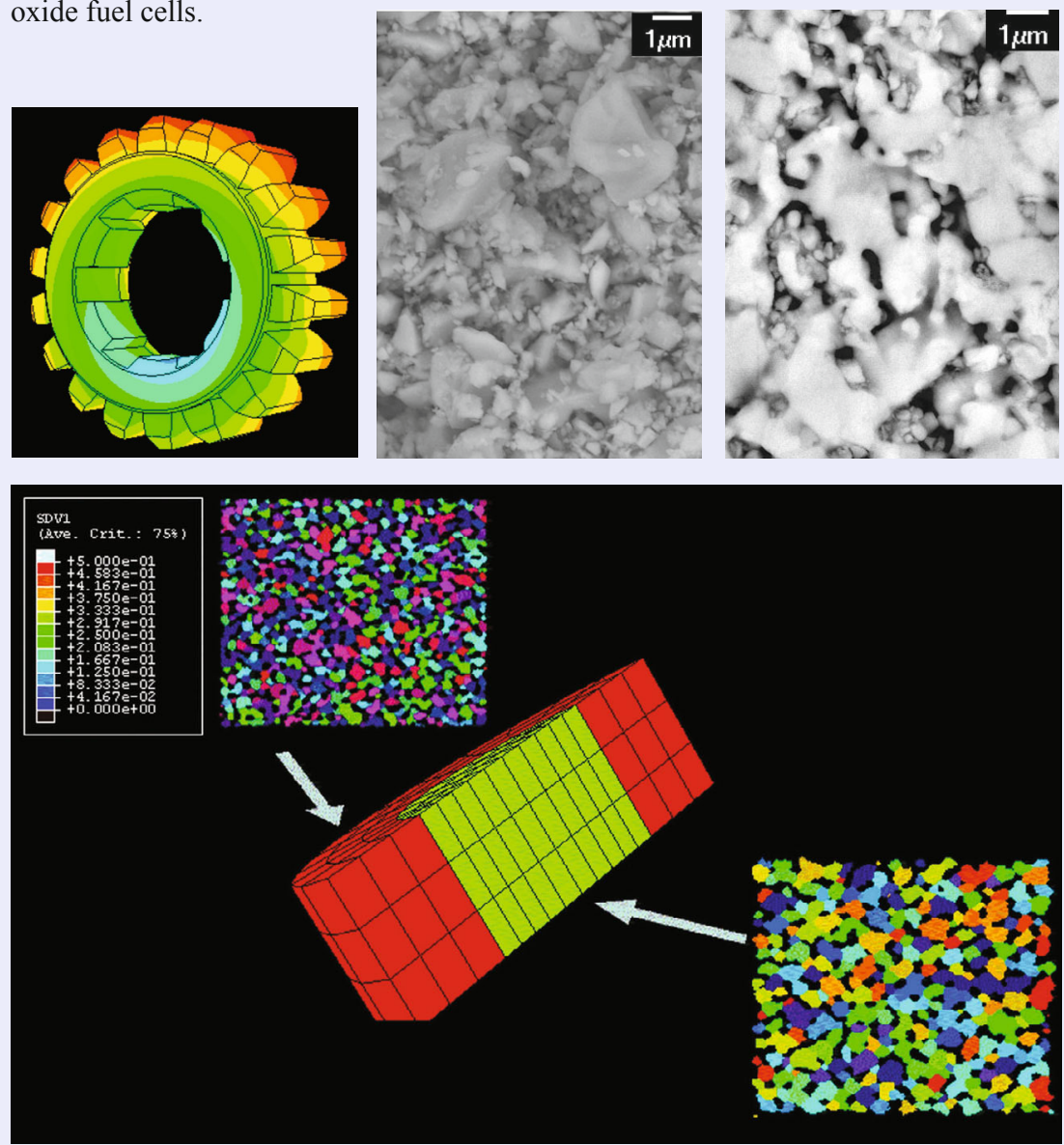

Figure B. Target part geometry, microstructure of 'green' and partially sintered parts, and multiscale simulation. (Credit: Haixia Shang, University of Washington; Aravind Mohanram, Saint-Gobain High Performance Materials; and Rajendra K. Bordia, Clemson University.) 
using many of the same commercial or open-source DFT-based tools mentioned previously (VASP, ABINIT, ${ }^{19}$ Quantum ESPRESSO ${ }^{20}$ ), each with extensive online support communities. At larger length scales, molecular dynamics, kinetic Monte Carlo, and finite element methods are common and often combine commercial packages, such as COMSOL, ${ }^{21}$ with homebuilt code. DFT methods work well for ground state properties such as electrode potentials, ferroelectric ordering, and spin-orbit coupling. A balance must still be struck between high throughput and high accuracy methods, since calculating, as an example, the critical temperature $T_{C}$ of a ferromagnet is still computationally quite expensive, and a significant challenge remains for calculating many properties simultaneously.

A number of DMREF teams are working to establish structure and property databases of electronic and

\section{DMREF Technical Highlight First-Principles Based Design of Spintronic Materials and Devices}

A team led by William Butler, University of Alabama, and Avik Ghosh, University of Virginia, is exploring the Full Heusler class of magnetic alloys in search of unique zero moment half-metals for use in magnetic tunnel junction devices. These alloys have the chemical formula $\mathrm{A}_{2} \mathrm{BC}$, where $\mathrm{A}$ and $\mathrm{B}$ are transition metals, but $\mathrm{C}$ does not have d-states near the Fermi energy (Figure C). An interesting feature of these alloys is their tendency to form Slater-Pauling half-metals. A Slater-Pauling half-metal (SPHM) is an alloy with a gap in the density of states for one of the spin channels which falls at the Fermi energy and in which the gapped spin channel holds precisely three electrons per atom. One simple consequence of the Slater-Pauling rule is that alloys that obey this rule and have 24 valence electrons per formula unit should have zero magnetic moment. These materials have no magnetic moment and so would not respond to moderate magnetic fields or generate demagnetization fields, yet they would be $100 \%$ spin polarized at the Fermi energy. The search for these unique materials has begun with a computational calculation of the electronic structure and lattice constants of 270 Full Heusler structures, and the team is now attempting to fabricate some highly promising candidates.
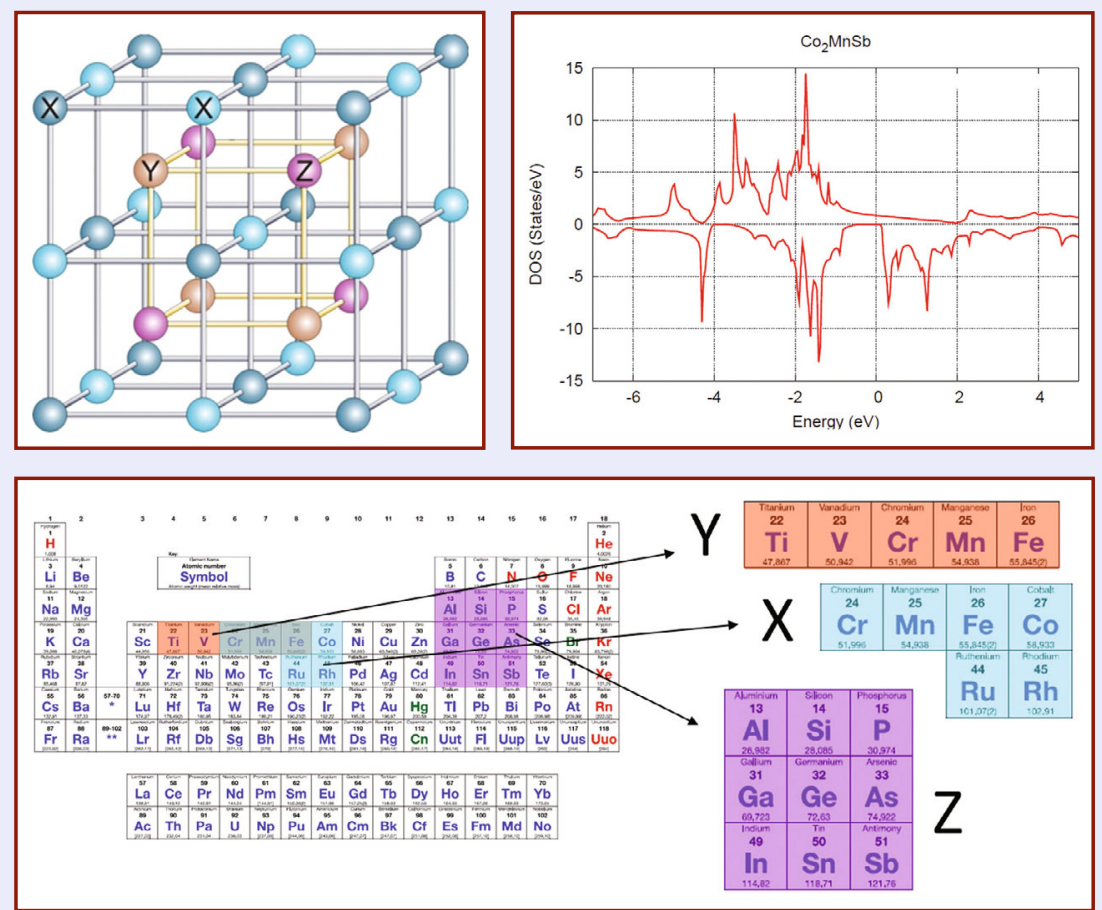

Figure C. Crystal structure of "Full Heusler" magnetic alloys and component elements within the periodic table, and density of states (DOS) for $\mathrm{Co}_{2} \mathrm{MnSb}$. (Credit: William Butler, University of Alabama.)

functional materials such as zeromoment half metals (see side bar), or strongly spin-orbit coupled materials using advanced computational tools. In each project, computational modeling is being employed to narrow the compositional field and select promising candidates, so that experimental efforts can focus on new synthesis and fabrication techniques. These unique synthesis techniques include:

- Use of high pressure to stabilize never-before observed oxynitride phases for applications in photocatalysis. (The team has previously made major methodological advances in computational modeling which have led to the discovery of novel materials, such as $\mathrm{Na}_{3} \mathrm{Cl}, \mathrm{NaCl}_{3}$, $\mathrm{MgO}_{2}$ and $\mathrm{Mg}_{3} \mathrm{O}_{2}$.)

- Precisely controlled atomic layer controlled growth within a molecular beam epitaxy system, required to synthesize interfacial structures with atomistic perfection (no crystal defects) yielding unique electronic properties

- Use of physical vapor deposition (PVD) to induce molecular alignment within organic glasses to induce anisotropies (birefringence and dichroism) that have been determined beneficial for organic light emitting diode (OLED) performance

\section{Soft Materials and Composites}

For the wide range of materials that encompasses synthetic polymers, to proteins and DNA, to organic glasses, computational tools are gaining a foothold in the research community, although significant challenges remain. Commercial software packages such as Gaussian ${ }^{22}$ and Materials Studio ${ }^{23}$ are available for modeling the electronic structure of small molecules and predicting basic physical properties. In some cases, their capabilities can be extended to calculate polymer properties. More typically, home-built codes based on 
molecular dynamics (at the atomic level) or self-consistent field theory (SCFT, for mesoscale behavior) are developed within specialized academic groups and do not transition into the workflow of the average researcher. Many tools also exist for predicting protein structure from primary sequence, yet they often require extensive computational resources or depend on solutions to structurally similar sequences. In general unlike crystalline solids, the tools to address soft materials problems are not predictiveparticularly for industrially relevant problems, in which the materials adopt highly non-equilibrium behavior over vast length and time scales. The possible exception to this is equilibrium block copolymer selfassembly for lithography applications.

Several DMREF projects are attempting to tackle major challenges of predictive computation in soft materials. The primary focus is on using computational methodsvalidated through advanced and novel experimental techniques - to understand the molecular mechanisms and environmental factors affecting self-assembly or crystal nucleation and growth, with the goal of "bottom up" engineering of material properties.

\section{The "Soft Issues": Challenges Faced and Opportunities Identified}

In addition to providing a forum for technical exchange, the NSFDMREF workshop was designed to highlight the barriers and opportunities specific to programs of a highly collaborative nature, as required to achieve the MGI-DMREF goals. Breakout discussions were catalyzed by the plenary lectures discussed earlier in this article and by presentations from NSF program managers, who highlighted programs that are available to support highly computational efforts. It was surprising to discover the commonality of issues faced by the various research teams, despite the fact that the technical topics are so widely varied.

\section{Cyberinfrastructure to Support Data Sharing and Software Development}

Many other scientific communities are recognizing the value of cyberenabled research. Comprehensive datasets, information processing, and high-powered computing enable researchers to ask largescale questions and accelerate research progress. As recognized with the announcement of MGI, the materials community is expected to greatly benefit from investments in cyberinfrastructure to facilitate and encourage collaboration, reuse of data, and advanced computation, as well as long-term digital preservation of data. The NSF-DMREF workshop presentations stressed that developments in cyberinfrastructure are important to both experimental (synchrotron data capture, storage, analysis, and curation) and explicitly computational (first principles calculations of complex structures and data mining of multidimensional spaces).

Discussions among the DMREF grantees during the workshop highlighted some significant challenges that the materials community - particularly at the level of fundamental researchfaces in implementing the MGI vision. They recognized the value in large-scale databases but noted that, in their own experiences, data sharing is primarily small-scale and informal. Implementing databases will require developing effective data standards and management. Measurement standards exist for some well-established procedures, but the grantees questioned how feasible such standards would be for leading edge research. They also pointed out the value in sharing both positive and negative results, yet it was acknowledged that there is little cultural impetus to do so at this time. Additionally, while data storage has become less expensive, effective mining of the broad and disparate types of materials datafrom two-dimensional microstructure images to multi-dimensional energy landscapes - and standardized metadata describing fabrication and/ or processing conditions is far from commonplace. Several grantees raised the concern of the cost of establishing and maintaining databases and the potential effect on research funding.

Some breakout discussions also centered on computational code development. Several typical strategies emerged among the DMREF grantees. These included use of commercially available codes, the addition of new features to publicly available packages, and the development of new software. Some agreed that a particular code will only survive beyond the tenure of a few students when it serves a dedicated non-academic population and provides them with practical solutions. Effective code development often requires collaborators with specific expertise, yet the grantees

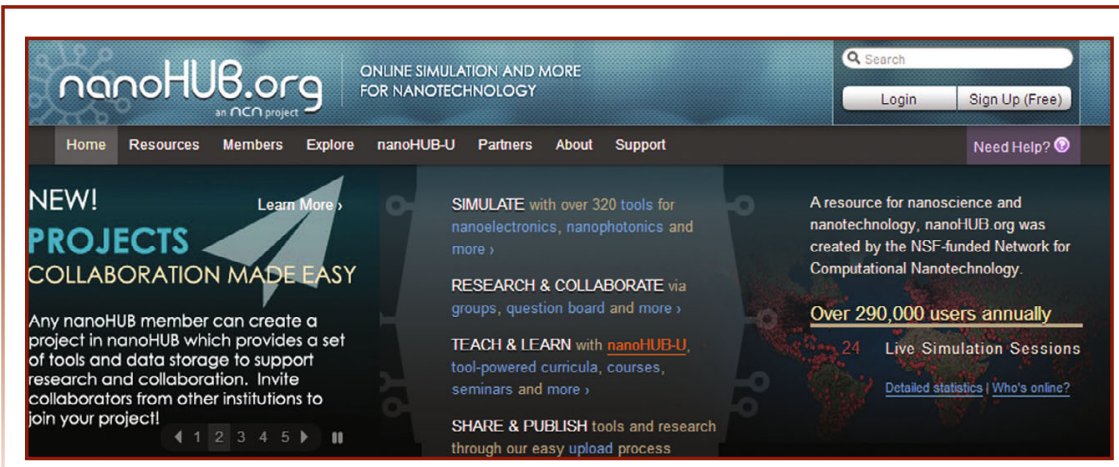

Figure 2: Screenshot of the nanoHUB homepage. nanoHUB can be accessed at nanohub.org. 


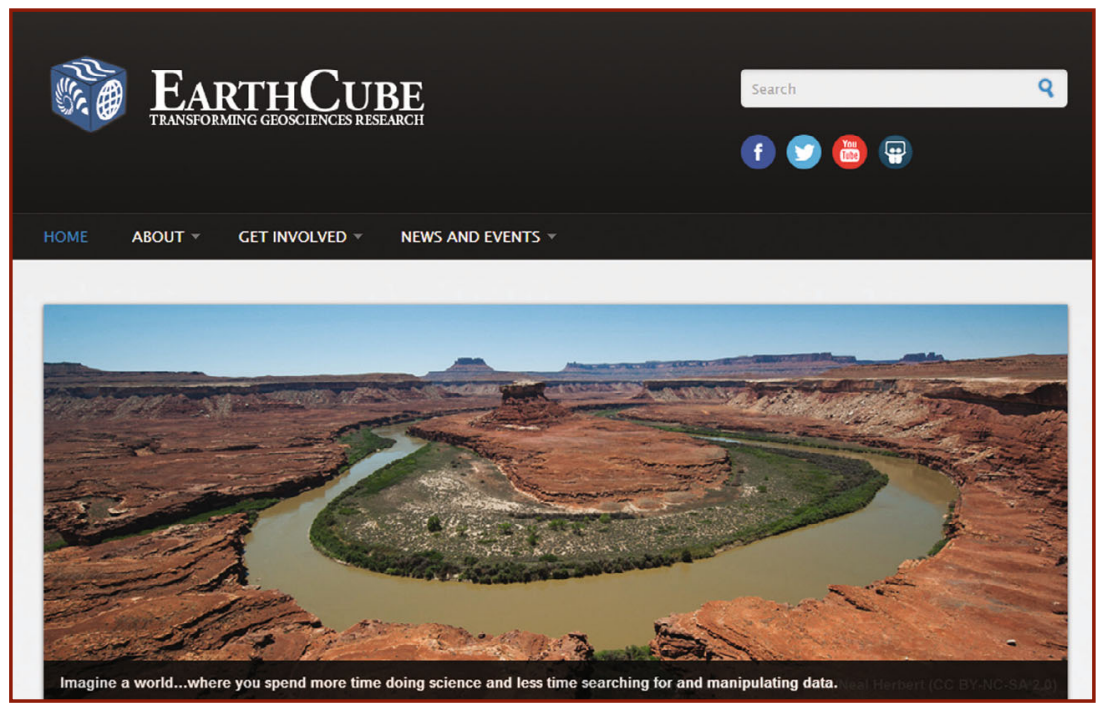

Figure 3: Screenshot of the EarthCube homepage. EarthCube can be accessed at www.earthcube.org.

felt there was little funding for such activity, and as a result, code is generally not sustained well.

The NSF has undertaken a number of efforts to support research communities in developing both broad and discipline-specific cyberinfrastructure. Some solutions demonstrated in existing programs overlap with the needs of materials researchers more than others, but they generally provide a base to begin framing the best path forward for the community. For example, the Network for Computational Nanotechnology and the associated website, nanoHUB ${ }^{24}$ (Figure 2), provide a free virtual meeting place and scientific cloud computing environment. It has rapidly grown since its inception in 2002, now hosting thousands of resources, including simulation tools, presentations, teaching materials, and courses. nanoHUB is powered by the HUBzero ${ }^{\circledR}$ platform, which has since served as a major cyberinfrastructure development resource for other scientific communities.

EarthCube $^{25}$ (Figure 3) represents a similar, but nascent effort, within the geosciences community. Despite the different research focus, the overlap in goals between MGI and EarthCube is substantial. EarthCube's vision is to change the modus operandi to one of integrating data and information across time scales, length scales, and research communities. In the few years of its existence, EarthCube leadership has recognized the primary importance of community engagement and worked hard to establish a solid social foundation on which to build its technical solutions.

The following links refer to other programs and resources to support both individual researchers and communities at various stages of cyberinfrastructure development:

- The Extreme Science and Engineering Discovery Environment (www.xsede.org /home) provides a virtual system of supercomputing resources and services to scientists at no cost.

- Open Science Grid (www .opensciencegrid.org) enables opportunistic usage and sharing of computational resources.

- The Computational and DataEnabled Science and Engineering program at NSF ( $w w w . n s f$ .gov/funding/pgm_summ .jsp?pims_id $=50 \overline{4813}$ ) funds new computational and data analysis approaches to enable scientific and engineering breakthroughs.

- The Software Infrastructure for Sustained Innovation program at NSF (www.nsf.gov/funding/pgm _summ.jsp?pims_id=504817) supports three classes of awards: Scientific Software Elements, Scientific Software Integration, and Scientific Software Innovation Institutes.

\section{Researcher Training}

The DMREF grantees represent a portion of a newly forming community of material discovery and development. Across the spectrum of materials development, the burdenand opportunity — of training the next generation of materials researchers falls disproportionately on the shoulders of individuals like them. In the eyes of many of the grantees, the factor that is presently slowing the MGI approach is not technical. Rather, it is identifying, recruiting, and training the next cohort of researchers.

The success of MGI requires “...a culture supporting the routine production and use of the tools developed." ${ }^{26}$ While coursework in computational methods is available at some schools, it is not yet a standard component of graduate education in materials science and engineering. Some educators are attempting to address the gap. For example, the University of Michigan Summer School for Integrated Computational Materials Education (ICME) aims to help others integrate computational techniques into their curricula. ${ }^{27}$

In breakout sessions during the workshop, the DMREF grantees pointed out that, to fully embrace the MGI approach to materials research, new courses, adaptation of existing curricula, and perhaps even new programs will need to be implemented so that students can be trained to carry out modern data analytics and data management. Interaction between materials science and computer science departments will need to be increased to provide knowledge and training in data informatics, as well as broaden the awareness of the particular challenges in managing materials data. Indeed, because such tight integration across disciplines is required to succeed, 
it is a requirement for proposals to the cyberinfrastructure programs discussed earlier in this article.

Even with enhanced curricula, DMREF and other interdisciplinary research grant programs provide the opportunity for focused technical education and training that would be otherwise difficult to achieve. Interdisciplinary work, by definition, takes place at the boundaries of traditional fields. Through collaborative projects, students can meaningfully and regularly interact with collaborators and students in the other group(s), giving them a deep understanding of not just of the results of a collaborator's work, but also how the data were generated and the nature of experimental or computational limitations.

Another approach some DMREF grantees have adopted is the organization of short summer schools as a way for students to actively participate and gain working knowledge in a new area. Chang-Beom Eom, University of Wisconsin-Madison, brought together students and professors in a two-day workshop, Multifunctional Interfacial Materials, in early 2013. Tresa Pollock, University of California, Santa Barbara, helped organize a two-week summer school, Materials in 3D: Modeling and Imaging at Multiple Length Scales, for advanced graduate students, postdocs, and early career faculty in 2013. Pollock is also organizing a winter workshop, High Temperature Materials and Coatings, to be held in early 2014. Janice Musfeldt, University of TennesseeKnoxville, David Vanderbilt, Rutgers University, and SangWook Cheong, Rutgers University, organized a week-long summer workshop, Enhanced Functionalities in 5d Transition-Metal Compounds from Large Spin-Orbit Coupling, in Telluride, Colorado in 2013. This workshop brought together theorists, computational scientists, and experimentalists working in this area.

\section{Conclusions}

In response to the call of the Materials Genome Initiative, the NSF established the DMREF program with the intent of supporting highly interdisciplinary, iterative research projects that push the boundaries of computation, experiment, and theory. Despite its relatively small size, the first cohort of grantees represents a broad swath of materials research.

The DMREF research projects are highly collaborative. Their early experiences highlight many challenges that the broader community faces in implementing the MGI vision. A lack of established cyberinfrastructure as well as the cultural resistance and logistical barriers to sharing data need to be overcome. Similarly, mechanisms for supporting long-term computational code development are currently lacking. In the eyes of many of the grantees, the key factor in slowing the MGI approach is identifying, recruiting, and training the next generation of researchers to perform this type of research. New courses in computational materials science, deepening collaborations with computer science departments, and more interdisciplinary training opportunities like the DMREF program are all needed.

Achieving the MGI's goals require engagement at all levels, from the "soft issues" of human development, to basic research, to product development. The DMREF program at NSF represents a promising first step at the discovery end of the continuum, and the opportunity for new projects will continue at least through 2014. (Researchers

\section{DMREF Technical Highlight \\ A Fundamental Approach to Study the Effect of Structural and Chemical Composition in Functionalized Graphene Materials \\ One team,in particular. is developing innovative multiscale experimental and computational techniques to advance graphene-based composites design. Led by Horacio Espinosa, Northwestern University, experiments are being conducted to characterize the nanoscale mechanical properties of graphene oxide-based structures. These experimental results, together with atomistic computations, will train coarse- grained models to simulate mesoscale composite systems comprised of multiple graphene oxide sheets interacting strongly with softer materials, such as polymer chains, block copolymers, and proteins (Figure D). Based on simulations to assess the role of interphase chemistries and geometric arrangements of constituents, coarse- grained models will provide design rules for specific composite properties such as strength and toughness. Within this bottom-up design of materials, microscale experiments validate optimal composition and structures. Through this approach, synergies between experimentation and computation will accelerate the design of novel composites.}

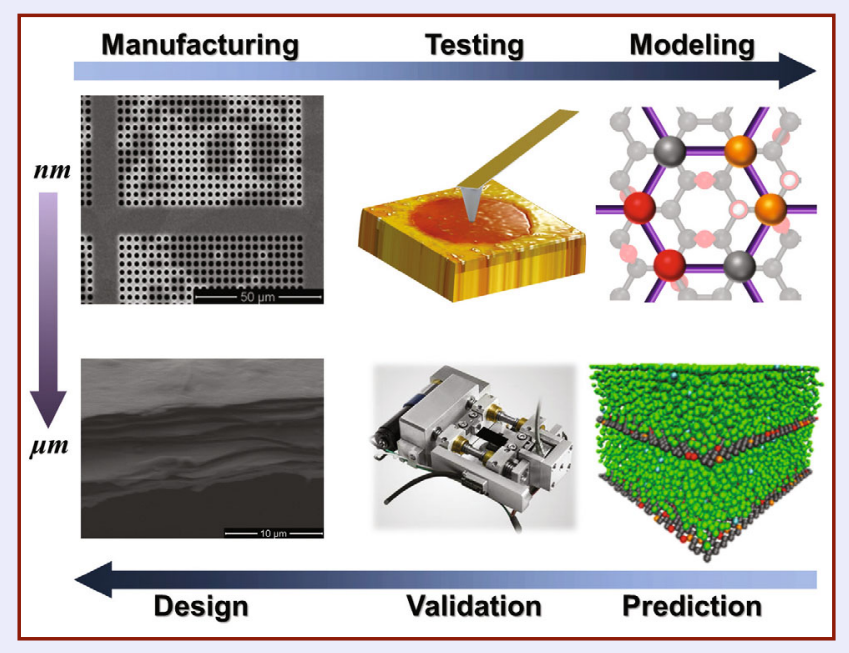

Figure D.

Scheme showing interrelation between modeling, experimental characterization, and manufacturing in functionalized graphene materials. (Credit: Horacio Espinosa, SonBinh Nguyen, Jiaxing Huang, and Sinan Keten, Northwestern University.) 


\section{DMREF Technical Highlight Programmable Peptide-Based Hybrid Materials}

Darrin Pochan, University of Delaware, and Jeffrey Saven, University of Pennsylvania, are leading a team to develop both predictive models and robust solution-based methods for assembling arrays of nanoparticles using custom polypeptides. While synthetic polymers typically assemble into relatively simple one- and two-dimensional structures, polypeptides offer the potential of highly sophisticated templates for controlling interparticle spacing and symmetry (Figure E), with applications such as enhanced light capture in photovoltaic cells. Through iterative feedback between probabilistic protein design methods, solution-based experimental assembly, and nano- to macro-scale characterization, the team is working to determine fundamental design principles and methods for non-natural polypeptide assemblies.

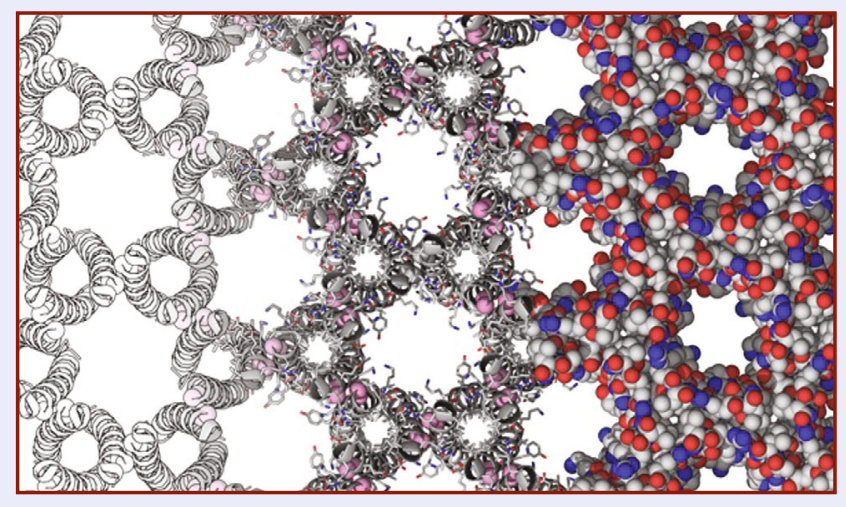

Figure E. Computationally designed P6-d model protein crystal. (Credit: Jeffery Saven, University of Pennsylvania.) interested in submitting proposals are encouraged to contact the relevant program officer listed in the Dear Colleague Letter posted at $w w w . n s f$ .gov/pubs/2014/nsf14020/nsf14020 .jsp.) Both the technical knowledge and broader experience gained through these projects will serve as important foundational pieces of the future materials development ecosystem.

The authors would like to thank the NSF for financial support of the DMREF Workshop through award number 1352571, with program oversight from Steve McKnight, Director, Division of Civil, Mechanical, and Manufacturing Innovation, and Mary Galvin, Director, Division of Materials Research. Thanks also to Mohsen Asle Zaeem, Missouri University of Science and Technology, and Leonid Zhigilei, University of Virginia, for helping to craft statements about phase field and molecular dynamics modeling, respectively. Similarly, Ram Seshadri, University of California,

\section{Acknowledgements}

Santa Barbara, and Vladan Stevanovic, National Renewable Energy Laboratory, are thanked for discussions of methods and challenges in functional materials, as is Glenn Fredrickson, University of California, Santa Barbara, for help with the statements on computation in soft materials.

\section{References}

1. Materials Genome Initiative (Washington, D.C.: The White House, 2011), www. whitehouse.gov/mgi. 2. Dear Colleague Letter - Designing Materials to Revolutionize and Engineer our Future (DMREF) (Arlington, VA: National Science Foundation, 2012), www.nsf.gov/pubs/2013/nsf13025/nsf13025.jsp. 3. Grant Opportunities for Academic Liaison with Industry (GOALI) (Arlington, VA: National Science Foundation), www.nsf.gov/funding/pgm_summ .jsp?pims_id=504699.

4. S.R. Niezgoda, A.K. Kanjarla, and S.R. Kalidindi, Integrating Materials and Manufacturing Innovation 2, 3 (2013).

5. Committee on Accelerating Technology Transition, National Research Council, "Accelerating Technology Transition: Bridging the Valley of Death for Materials lopenbook.php?record_id=11108.

6. Vienna Ab initio Simulation Package (Vienna, Austria: Computational Materials Physics, 2012), www.vasp.at.

7. Full Potential Linearized Augmented Plane Wave Method (FLAPW-Method) (Julich, Germany: Forschungszentrum Jülich $\mathrm{GmbH}, 2011$ ), www.flapw.de and Processes in Defense Systems" (Washington, D.C.: National Academies Press, 2004), www.nap.edu /pm/index.php.

8. Z.T.Y. Liu, X. Zhou, S.V. Khare, and D. Gall, J. Phys.: Condens. Matter 26, 025404 (5 December 2013).

9. Thermo-Calc Software (McMurry, PA: Thermo-Calc Software Inc.), www.thermocalc.com/.

10. Pandat (CompuTherm LLC, Madison, WI), www .computherm.com/.

11. FACTSage (Thermfact/CRCT, Montreal, Canada), www.factsage.com/.

12. Large-scale Atomic/Molecular Massively Parallel Simulator (LAMMPS) (Sandia National Laboratories, Albuquerque, NM), lammps.sandia.gov/.

13. "Integrated Computational Materials Engineering" (Washington, D.C.: National Academy of Engineering, 2008).

14. Microstructure Evolution Simulation Software (MICRESS) (ACCESS, Aachen, Germany), web .access.rwth-aachen.de/MICRESS/.

15. Parallel Dislocation Simulator (ParaDIS) (Lawrence Livermore National Laboratory, Livermore, CA), paradis.stanford.edu/.

16. VPSC (ViscoPlastic Self-Consistent) Code (maintained by Ricardo A. Lebensohn, Los Alamos, NM), public.lanl.gov/lebenso/.

17. "User Material Subroutine for Single Crystal Plasticity," www.columbia.edu/ jk2079/Kysar _Research_Laboratory/Single_Crystal_UMAT.html. 18. J. M. Rondinelli, N.A. Benedek, D.E. Freedman, A. Kavner, E.E. Rodriguez, E.S. Toberer, and L.W. Martin, Amer. Ceram. Soc. Bull., 92 (2) (December 2013), p. 14.

19. ABINIT (The ABINIT Group, Université Catholique de Louvain, Louvain-la-Neuve, Belgium), www.abinit

. org/.

20. Quantum ESPRESSO (opEn-Source Package for Research in Electronic Structure, Simulation, and Optimization) (Quantum ESPRESSO Foundation, London), www.quantum-espresso.org/.

21. COMSOL (COMSOL, Inc., Burlington,MA), www .comsol.com.

22. Gaussian software (Gaussian, Inc., Wallingford, CT), www.gaussian.com/.

23. Materials Solution software (Accelrys, Inc., San Diego, CA), accelrys.com/products/materials-studio/. 24. nanoHUB (Purdue University, West Lafayette, IN), nanohub.org/.

25. EarthCube (National Science Foundation, Arlington, VA), www.earthcube.org/.

26. "Materials Genome Iniaiative for Global Competitiveness" (National Science and Technology Council, Washington, D.C.), www.whitehouse.gov/sites/default/ files/microsites/ostp/materials_genome_initiative-final .pdf.

27. Lynne Robinson, "Short Course Organizer Takes the Long View on ICME Implementation" Tech News Headlines (Warrendale, PA: TMS, 3 March 2013), www.tms.org/pubs/journals/JOM/techHeadlinesArticle .aspx?articlel $D=4940$. Kathleen Feldman is the AAAS Science
and Technology Policy Fellow, Civil,
Mechanical, and Manufacturing Innovation,
Directorate for Engineering, National
Science Foundation, and can be contacted
at kfeldman @ nsf.gov. Sean R. Agnew is
the Heinz and Doris Wilsdorf Associate
Professor of Materials
Science and Engineering,
University of Virginia, and
can be contacted at agnew@
virginia.edu. 